\title{
Bedside peritoneal drainage: A primary treatment for perforated necrotizing enterocolitis
}

\author{
T Gobran, MD; M Khalifa, MD; A Waly, MD; Salah M AbdElAal, MD; \\ Kamal abd El-elah Aly, $M D$
}

Department of Pediatric Surgery, Zagazig and Mansoura Universities, Egypt.

\begin{abstract}
Background and purpose: Necrotizing enterocolitis is one of the leading causes of morbidity and mortality in neonatal intensive care units particularly in places without neonatal surgical facilities. The best treatment for perforated necrotizing enterocolitis is uncertain. Bedside peritoneal drainage has been used as primary treatment in the management of perforated necrotizing enterocolitis. This study aimed to compare outcomes of bedside peritoneal drainage done by general surgeons as a primary procedure for the treatment of preterm and/or low birth weight neonates with perforated necrotizing enterocolitis to outcomes of early laparotomy performed by pediatric surgeons as regards effectiveness of the procedure as a definitive treatment, the need for delayed laparotomy and mortality rate.

Patients and methods: Fifty cases of preterm and/or low birth weight neonates with perforated necrotizing enterocolitis were randomly assigned to one of two groups. Group I was managed by bedside peritoneal drainage done by general surgeon while laparotomy was reserved for non responding cases. Group II was managed by early laparotomy done by pediatric surgeon. Data collected from all cases included birth weight (g), gestational age (weeks), gender, age at operation (bedside peritoneal drainage or early laparotomy). Delayed laparotomy was performed for infants who developed persistent fecal fistula or suffered late intestinal stricture as well as for closure of enterostomy. Outcomes of bedside peritoneal drainage and early laparotomy were recorded and statistically compared regarding the effectiveness of procedure as a definitive treatment, need for delayed laparotomy and mortality.

Results: 16 cases (64\%) showed clinical improvement after bedside peritoneal drainage. Bedside peritoneal drainage in Group I and early laparotomy in Group II were effective as a definitive treatment in 10 cases (40\%) and 15 cases (60\%) respectively. Delayed laparotomy was indicated in 7 cases (28\%) in Group I and 8 cases (32\%) in Group II. Mortality was recorded in 8 patients (32\%) in BPD group and in 9 patients (36\%) in laparotomy group.

Conclusion: According to this study, outcomes of bedside peritoneal drainage as a primary treatment for low birth weight and/or preterm neonates with perforated necrotizing enterocolitis showed no significant statistical difference as regards the need for delayed laparotomy and mortality rate when compared to the outcomes of early laparotomy as a primary treatment for the same conditions. Bedside peritoneal drainage provides a useful primary procedure for the management of preterm and low birth weight neonates with perforated necrotizing enterocolitis particularly in healthcare facilities without neonatal surgery capacity.
\end{abstract}

Key words: Necrotizing enterocolitis, peritoneal drainage, neonatal intestinal perforation.

\section{Introduction:}

Necrotizing enterocolitis (NEC) is the most common gastrointestinal emergency that affects the newborn. ${ }^{1}$ A total of $90 \%$ of NEC cases occur in premature infants. ${ }^{2} \mathrm{NEC}$ is one of the leading causes of morbidity and mortality in neonatal intensive care units (NICU). 3,4 Over the last two decades the mortality rate from NEC remained steady at $20 \%-50 \%$ even in developed countries. 5,6 
Although pathogenesis of the disease is not fully understood, in severe cases, NEC rapidly progresses from bacterial invasion of the intestinal wall to full-thickness bowel necrosis, leading to perforation and subsequent peritonitis, sepsis, and possibly death. ${ }^{7}$

Traditional surgical management of neonatal bowel perforation secondary to NEC has been based on established surgical principles of laparotomy (LAP), resection of necrotic bowel, debridement, and exteriorization. ${ }^{8}$ In 1977 , Ein et $\mathrm{al}^{9}$ recommended bedside peritoneal drainage (BPD) as a "temporizing" procedure for perforated NEC in very low birth weight neonates. This operation was designed for patients considered too unstable to undergo LAP. Since the introduction of BPD, there have been several reports which suggested that BPD may serve as a definitive therapy. In many centers, BPD has become the routine approach regardless of severity of the underlying illness. ${ }^{10,11}$ Some reports challenge the role of LAP as an initial choice of surgery and suggest that bowel perforation secondary to NEC needs a far less invasive surgical approach for adequate initial management. 10,12

In our region (Sharkeya and Mansoura Governerates- Egypt) there is a single pediatric surgery unit in each governate. Although these units serve a big region with heavy population, they suffer limited resources. Frequently, there is lack of beds and unfeasibility to accept cases with perforated NEC. These factors in addition to high mortality rate of cases with perforated NEC and the challenge of optimal surgical treatment prompted us to inquire about the value of BPD performed by general surgeons as a primary treatment for neonates with perforated NEC.

\section{Purpose:}

This work aimed to compare outcomes of bedside peritoneal drainage done by general surgeons as a primary procedure for the treatment of preterm and/or low birth weight neonates with perforated necrotizing enterocolitis to outcomes of early laparotomy performed by pediatric surgeons as regards effectiveness of the procedure as a definitive treatment, the need for delayed laparotomy and mortality rate.

\section{Patients and methods:}

This randomized clinical study was conducted in NICU-Faculty of MedicineZagazig and Mansoura Universities in the period from January 2007 to June 2010. It included 50 cases of preterm and/or low birth weight (LBW) neonates with perforated NEC. Patients were randomly assigned to one of two groups:

Group I (25 cases): underwent BPD as a primary treatment done by general surgeon. Early laparotomy was reserved for patient who showed no or limited response to BPD.

Group II (25 cases): underwent early laparotomy performed by pediatric surgeon.

Criteria for exclusion of patients from this study included full term patients, normal birth weight, associated anomalies and parent's refusal.

Diagnosis of intestinal perforation was confirmed by plain X-ray of the abdomen that revealed free air in the peritoneal cavity (pneumoperitoneum).

Parents were counseled and asked by the attending surgeon to provide written informed consent.

BPD was performed in NICU under complete sterile conditions by general surgeon. Using local anaesthesia, full thickness incision $(0.5 \mathrm{~cm})$ was made in the right or left lower quadrant of the abdomen and the peritoneal cavity was entered under direct vision. A $10 \mathrm{~F}$ catheter was inserted carefully into peritoneal cavity, and peritoneal fluid was collected for microbiologic cultures. The catheter was fixed at the skin and the end left free in a collection bag. The peritoneal cavity was then irrigated with warm saline solution until retrieved fluid became clear. If the peritoneal cavity was believed to be inadequately drained, as evidenced by re-accumulation of air or fluid in the abdomen, the original drain was manipulated or an additional drain was inserted to help better peritoneal drainage.

Cases randomly assigned to early LAP underwent abdominal exploration through a transverse abdominal incision. Necrotic intestine was resected. Intestinal stomas or primary anastomosis was performed depending on site, extent of intestinal necrosis and general condition of patients. Evidence of further intestinal necrosis or perforation was the 
indication for additional early laparotomies.

The patients were closely monitored in NICU and reassessed by surgeons every 12 to 24 hours. Afterward management of group I depended on the clinical course of the patients as follows:

1. Clinical improvement and stability: It meant haemodynamic stabilization, decrease abdominal distension, decrease abdominal wall cellulitis, no palpable mass, less painful abdominal palpation and no further pneumoperitoneum. In these situations, the peritoneal drain was removed when it no longer discharged fluid or after the development of a fecal fistula.

2. Clinical deterioration: It was based on the joint clinical judgment of the attending surgeons. It meant increased abdominal distension and/or discoloration, persistent or increasing pneumoperitoneum, palpable mass, signs of persistent intestinal obstruction and no passage of stools. In these situations, early LAP was performed within 12 to 24 hours from insertion of peritoneal drain.

Delayed LAP was performed when the patient developed persistent fecal fistula or suffered late intestinal stricture proved clinically and radiologically.

Data Collected from all cases included birth weight (g), gestational age (weeks), gender, and age at operation (BPD or early LAP). Routine data recorded at NICU included medications given at onset of NEC or perforation, need for mechanical ventilation, $\mathrm{PaO}_{2} / \mathrm{FiO}_{2}$, urine output $(\mathrm{ml} / \mathrm{Kg} /$ hour) and mean arterial pressure.

The outcome variables in this study were the need for delayed LAP and mortality rate hence to compare the outcome of BPD versus early LAP as the primary treatment for perforated NEC in preterm and/or LBW neonates.

Definitive treatment was defined in this work as the treatment that resulted in improvement of the patient clinical condition, recovery of normal gastrointestinal function and normal enteral feeding.

\section{Data management:}

Data were collected, tabulated and finally analyzed using Epi-6 and Statistical Package for Social Science (SPSS) version 19 statistical programs. Descriptive statistics were used such as percentage, arithmetic mean and standard deviation.

Statistical tests of significance were used to compare between the studied groups as Chisquare test and t-test. $\mathrm{P}$ value $\leq 0.05$ was considered significant difference and $\mathrm{P}$ value $\leq 0.01$ was considered highly significant difference.

\section{Results:}

This study included 29 boys (58\%) and 21 girls $(42 \%)$. The mean gestational age was 30.6 weeks and 31.4 weeks in group I and group II respectively. The mean birth weight was 1280.9 grams and 1290.3 grams in groups I and II respectively. The mean Apgar score at 1 minute and 5 minutes was 5.4 and 7.2 for group I and 6.3 and 8.5 for group II. The mean age at operation was 13.3 days and 13.7 days in group I and group II respectively Table(1).

In group I the range of peritoneal drain duration after excluding the cases that required early LAP was $10-14$ days with a mean of 12.6 days.

In group I 16 cases $(64 \%)$ showed initial clinical improvement after BPD. Clinical deterioration occurred in 3 cases while clinical improvement was limited in 6 cases. These 9 cases underwent early LAP (36\%). Three cases needed intestinal resection and with primary anastomosis and 6 cases ended with enterostomies.

Eight cases died in group I with mortality rate of $32 \%$. Five cases died after early LAP because of massive NEC, 2 cases of unknown cause and 1 case because of type IV intraventricular haemorrage. Seven cases needed delayed LAP (28\%) for intestinal stricture in 3 cases, fecal fistula in 3 cases and one case for closure of stoma. BPD was effective as a definitive treatment in 10 cases (40\%) after excluding 3 cases that died after initial clinical improvement and 3 cases who developed persistent fecal fistula.

In group II early LAP and damaged intestinal resection was performed followed by primary intestinal anastomosis in 17 cases and diverting stoma in 8 cases. Nine cases died with mortality rate of $36 \%, 2$ of them were intraoperative and 7 cases were during early postoperative course. Eight cases required 
delayed LAP (32\%), Table(2). It was indicated in 5 cases for closure of stoma, 2 cases due to intestinal stricture and 1 case due to development of fecal fistula. Early LAP was effective as a definitive treatment in 15 cases (60\%) after excluding one case that developed early postoperative fecal fistula. No cases in this group required further early laparotomies.
In group I early LAP was indicated for 9 cases $(9 / 25)$ because of no or limited response to BPD. When we compared that to performing early LAP as the primary treatment in all cases of group II, we found statistically significant privilege to BPD.

Table (1): Demographic data of the cases included in the study.

\begin{tabular}{|l|c|c|c|c|}
\hline Items & $\begin{array}{c}\text { Group I } \\
\text { Mean } \pm \text { SD }\end{array}$ & $\begin{array}{c}\text { Group II } \\
\text { Mean } \pm \text { SD }\end{array}$ & t test & P value \\
\hline Mean gestational age (Weeks) & $30.6 \pm 2.3$ & $31.2 \pm 3.2$ & 0.76 & 0.45 \\
\hline Mean birth weight (Grams) & $1280.9 \pm 50.6$ & $1285.3 \pm 35.7$ & 0.36 & 0.7 \\
\hline Mean Apgar score & & & & \\
1 minute & $5.4 \pm 1.5$ & $6.3 \pm 1.8$ & 1.9 & 0.06 \\
5 minutes & $7.2 \pm 2.2$ & $8.5 \pm 2.5$ & 1.95 & 0.06 \\
\hline Mean age at operation (Days) & $13.3 \pm 3.3$ & $13.7 \pm 3.8$ & 0.4 & 0.7 \\
\hline
\end{tabular}

Table (2): Treatment outcomes of the study groups.

\begin{tabular}{|c|c|c|c|c|}
\hline Items & $\begin{array}{c}\text { Group I } \\
\text { BPD } \\
(\mathrm{n}=25)\end{array}$ & $\begin{array}{c}\text { Group II } \\
\text { LAP } \\
(\mathrm{n}=25)\end{array}$ & $\begin{array}{c}\text { Chi-square } \\
(\mathrm{X} 2)\end{array}$ & P value \\
\hline Definitive treatment & $10(40 \%)$ & $15(60 \%)$ & 2 & 0.16 \\
\hline Early LAP & $9(36 \%)$ & $25(100 \%)$ & 23.5 & $<0.001$ \\
\hline Delayed LAP & $7(28 \%)$ & $8(32 \%)$ & 0.1 & 0.76 \\
\hline Mortality & $8(32 \%)$ & $9(36 \%)$ & 0.09 & 0.8 \\
\hline
\end{tabular}

In this study 5 cases died out of 9 who underwent early LAP because of no or limited response to BPD. When this result was compared to mortality after BPD only $(3 / 16)$ we found that the difference was statistically insignificant $\left(\mathrm{X}^{2}=3.6\right.$ and $\mathrm{P}$ value $\left.=0.06\right)$. When mortality after BPD and early LAP was compared to that after early LAP only $(9 / 25)$, the difference was statistically insignificant, $\left(\mathrm{X}^{2}=1.04\right.$ and $\mathrm{P}$ value $\left.=0.3\right)$.

\section{Discussion:}

Despite decades of clinical advances and research, the management of NEC continues to offer a considerable challenge to the pediatric surgeon. ${ }^{13,14}$ Exploratory LAP or peritoneal drainage, which is the favorable choice as a primary treatment for perforated NEC in neonates? The answer for this question has been controversial.

The treatment of perforated NEC by exploratory LAP carries high significant risk in preterm and LBW neonates. ${ }^{8}$ On the other hand, effective BPD will hypothetically draw off peritoneal gas, pus, and stool, thus minimizing operative stress and avoiding intestinal resection. This makes BPD an attractive alternative to LAP. ${ }^{9}$ Moreover, BPD has advantages of being bed side procedure, cheap, technically easy, does not need operating room, or general anesthesia, and may not entail stoma or second operation. Nevertheless, 
evaluation of disease process and extent of bowel involvement is incomplete by this approach. ${ }^{15}$

Several regions in Egypt suffer lack of pediatric surgery facilities. Pediatric Surgery Unit of either Zagazig or Mansoura Universities is the sole neonatal surgical unit in their region with limited resources and inability to accept all referred cases of perforated NEC from NICUs of other hospitals. It is even difficult sometimes to reach the patients at distant hospitals. For this reason this study has emerged as a trial to study if there is a possibility of using BPD performed by general surgeon as a primary treatment for perforated NEC in these hospitals aiming to save those neonates.

In this study a group of preterm and LBW neonates who suffered perforated NEC were managed by BPD done by general surgeons at our hospital as a primary treatment. Effectiveness of this procedure as a definitive treatment, the need for delayed LAP and mortality were compared to another group of similar patients with perforated NEC who were managed primarily by early LAP performed by pediatric surgeons.

Although many studies have reported the outcomes of treatment with BPD or LAP, these reports contain significant bias influencing the patient selection and it is not possible, even through meta-analysis, to determine whether BPD or LAP is the better technique for any size infant. ${ }^{10,16}$ In the present work, statistical testing of demographic data of both groups proved no statistically significant difference between both groups' criteria, which supports unbiased patient selection in the study.

Ein and colleagues argue that LAP with intestinal resection is associated with high rates of morbidity and mortality in very LBW infants. Therefore, they recommend BPD as the preferred initial procedure for neonates with perforated NEC. 9

BPD procedure was initially introduced as a method for pre-operative resuscitation of critically ill infants with complicated NEC. However, sometimes it was used as the definitive procedure for treatment of neonates with perforated NEC. 17,18

Several authors have advised BPD as a "temporizing procedure", for all children weighing less than 1500 grams and for unstable babies more than 1500 grams, followed by LAP in two to three days. ${ }^{19-22}$

$\mathrm{BPD}$ is preferred by some surgeons because they are cautious against the risk of waiting too long before performing a LAP. Dimmitt and colleagues suggest that death may be avoided by performing a timely LAP. ${ }^{24}$ Takamatsu and colleagues have recommended BPD as definitive strategy for a selected group of extremely LBW babies. ${ }^{25}$ In this study early LAP was performed if the patients did not show clinical improvement within 12-24 hours of BPD.

In 1990 Ein and colleagues ${ }^{19}$ presented their 13-year experience with BPD in 37 patients with bowel perforation secondary to NEC. Sixty-five percent of these patients weighted less than 1000 grams and $88 \%$ less than 1500 grams. One third of the reported patients recovered completely by BPD. In the remaining patients: nine (24\%) died rapidly before LAP, nine $(24 \%)$ underwent an early LAP (within 24 hours), and 7 (22\%) underwent a delayed LAP for bowel obstruction or fistula formation.

Dimmitt and colleagues treated 26 patients with perforated NEC. Nine cases were treated with LAP and 17 with BPD. Survival rate was similar between LAP and BPD. Four patients in the BPD group underwent salvage LAP for perceived clinical deterioration. All of these patients died. The clinical status of patients who had salvages LAP and died was similar to those who did not and lived. ${ }^{24}$

By reviewing previous studies it is noted that there is wide variation in the results of BPD regarding survival and outcome. Some reports suggested that $B P D$ resulted in the unexpected survival of the infants with perforated NEC, with survival rates approaching or exceeding those with LAP, ${ }^{19,25}$ while others suggested that LAP was the superior treatment. ${ }^{23,15}$

In this work mortality rates showed statistically insignificant difference between the group treated primarily with BPD, where LAP was reserved for cases that showed no or limited response, and the group treated primarily with exploratory LAP. Thus BPD in our view provides a reasonable option for healthcare facilities devoid of pediatric surgery capacity provided the attending physician is trained to perform BPD in neonates. 
In this study early LAP was indicated in 9 cases of Group I (9/25) because of no or limited response to BPD. When that was compared to performing LAP as the primary treatment for all cases of Group II, we found statistically highly significant privilege to BPD. This result supports the role of BPD as primary procedure for neonates with perforated NEC and unfavorable conditions for LAP. LAP can hence be reserved for the cases not responding to BPD which will help saving neonates lives as well as healthcare resources. This is supported by Lessin et al recommendation to use BPD as initial management of all LBW infants with complicated NEC before definitive LAP. 16,26

However, there is a published literature that suggests that peritoneal drainage can be a satisfactory definitive procedure, particularly in the very LBW infant. Morgan, et al mentioned that BPD alone provided definitive surgical intervention in $74 \%$ of cases with complicated NEC. ${ }^{22}$

An international multicenter randomized controlled trial was performed between 2002 and 2006. Sixty-nine patients were randomized (35 drain, 34 LAP). Early LAP was performed in 26/35 (74\%) patients. BPD was effective as a definitive treatment in only $4 / 35(11 \%)$ surviving neonates. ${ }^{27}$

Romero et al, on their study of 13 cases of perforated NEC ( 6 in drainage group and 7 in LAP group) concluded that BPD is a temporary stabilizing procedure and could not be considered as a definitive surgical treatment. ${ }^{28}$

In our study BPD was effective as a definitive treatment in 10 cases $(40 \%)$. The explanation of the wide discrepancy of our result from the previously mentioned studies is the difficulty to differentiate between perforated NEC and isolated bowel perforation based on clinical and radiological findings. There are studies that seem to indicate that BPD is more advantageous in neonates with isolated perforations of the gastro-intestinal tract not related to NEC. For neonates with perforation caused by NEC, peritoneal drainage may provide temporary stabilization, but most of these infants require subsequent LAP, and few survive. ${ }^{29}$

One multicentre prospective study reported the overall incidence of postoperative intestinal stricture at $10.3 \%$ and no difference between the initial LAP versus the initial BPD groups. ${ }^{30}$ However other studies suggest that strictures occur more frequently in patients who have undergone BPD. 5

In this study, intestinal stricture occurred in 4 cases $(16 \%)$ of BPD group and 2 cases $(8 \%)$ of LAP group. A possible explanation is that the damaged areas which are prone to stricture formation upon healing would have been resected at the time of LAP.

\section{Conclusion:}

According to this study, outcomes of bedside peritoneal drainage as a primary treatment for low birth weight and/or preterm neonates with perforated necrotizing enterocolitis showed no significant statistical difference as regards the need for delayed laparotomy and mortality rate when compared to the outcomes of early laparotomy as a primary treatment for the same conditions. Bedside peritoneal drainage provides a useful primary procedure for the management of preterm and low birth weight neonates with perforated necrotizing enterocolitis particularly in healthcare facilities without neonatal surgery capacity.

\section{Recommendation:}

BPD is an easy, cheap and affordable option for stabilizing neonates with perforated NEC. We recommend BPD training for general surgeons and pediatricians in NICU to help saving neonates with perforated NEC in health care centers without pediatric surgery facility.

\section{References:}

1- Stoll BJ: Epidemiology of necrotizing enterocolitis. Clin Perinatol 1994; 21: 205 218.

2- Maayan-Metzger A, Itzchak A, Mazkereth $\mathrm{R}$, Kuint J: Necrotizing enterocolitis in fullterm infants: Case-control study and review of the literature. J Perinatol 2004; 24: 494499.

3- Hsueh W, Caplan MS, Qu XW, et al: Neonatal necrotizing enterocolitis: Clinical considerations and pathogenetic concepts. Pediatr Dev Pathol 2003; 6: 6-23.

4- Amoury RA: Necrotizing enterocolitis - a continuing problem in the neonate. World J Surg 1993; 17: 363-373. 
5- Horwitz JR, Lally KP, Cheu HW, Vazquez WD, Grosfeld JL, Ziegler MM: Complications after surgical intervention for necrotizing enterocolitis: A multicenter review. J Pediatr Surg 1995; 30(7): 994998; discussion 998-999.

6- Bisquera JA, Cooper TR, Berseth CL: Impact of necrotizing enterocolitis on length of stay and hospital charges in very low birth weight infants. Pediatrics 2002; 109(3): 423-428.

7- Kliegman RM, Fanaroff AA: Necrotizing enterocolitis. N Engl J Med 1984; 310: 1093-1103.

8- Sharma R, Tepas J J 3 ${ }^{\text {rd }}$, Mollitt DL, Pieper P, Jacksonville PW: Surgical management of bowel perforations and outcome in very low-birth-weight infants $(<1,200 \mathrm{~g}) . J$ Pediatr Surg 2004; 39(2): 190-194.

9- Ein SH, Marshall DG, Gervan D: Peritoneal drainage under local anesthesia for perforations from necrotizing enterocolitis. J Pediatr Surg 1977; 12: 963-967.

10-Rovin JD, Rodgers BM, Burns RC, et al: The role of peritoneal drainage for intestinal perforation in infants with and without necrotizing enterocolitis. J Pediatr Surg 1999; 34: 143-147.

11-Bysiek A, Palka J, Pietrzyk JJ, et al: Peritoneal drainage as an alternative to laparotomy in premature infants with complicated necrotizing enterocolitis. Przeg Lek 2002; 59: 67-79.

12-Demestre X, Ginovart G, Figueras-Aloy J, et al: Peritoneal drainage as primary management in necrotizing enterocolitis: A prospective study. $J$ Pediatr Surg 2002; 37: 963-967.

13-Kurscheid T, Holschneider AM: Necrotizing enterocolitis (NEC) - mortality and longterm results. Eur J Pediatr Surg 1993; 3(3): 139-143.

14-Patel JC, Tepas JJ, 3 ${ }^{\text {rd }}$, Huffman SD, Evans JS: Neonatal necrotizing enterocolitis: The long-term perspective. Am Surg 1998; 64(6): 575-579.

15-Ehrlich PF, Sato T T, Short B L, Hartman $\mathrm{G} \mathrm{E}$ : Outcome of perforated necrotizing enterocolitis in very low birth weight neonate my be independent of the type of surgical treatment. Am Surg 2001; 67: 752756.
16-Lessin MS, Luks FI, Wesselhoeft CW: Peritoneal drainage as definitive treatment for intestinal perforation in infants with extremely low birth weight ( $<750$ gms). J Pediatr Surg 1998; 33: 370-372.

17-Albanese CT, Rowe MI: Necrotizing enterocolitis. Semin Pediatr Surg 1995; 4(4): 200-206.

18-Holman RC, Stehr-Green JK, Zelasky MT: Necrotizin enterocolitis mortality in the United States, 1979-85. Am J Public Hlth 1989; 79(8): 987-989.

19-Ein SH, Shandling B, Wesson D, Filler RM: A 13-year experience with peritoneal drainage under local anesthesia for necrotizing enterocolitis perforation. J Pediatr Surg 1990; 25(10): 1034-1036.

20-Ahmed T, Ein S, Moore A: The role of peritoneal drains in treatment of perforated necrotizing enterocolitis: Recommendations from recent experience. J Pediatr Surg 1998; 33(10): 1468-1470.

21-Cheu HW, Sukarochana K, Lloyd DA: Peritoneal drainage for necrotizing enterocolitis. J Pediatr Surg 1988; 23: 557561.

22-Morgan LJ, Shochat SJ, Hartman GE: Peritoneal drainage as primary management of perforated NEC in the very low birth weight infant. J Pediatr Surg 1994; 29: 3034.

23-Azarow KS, Ein SH, Shandling B, Wesson D, Superina R, Filler RM: Laparotomy or drain for perforated necrotizing enterocolitis: Who gets what and why? Pediatr Surg Int 1997; 12: 137-139.

24-Dimmitt RA, Meier AH, Skarsgard ED, et al: Salvage laparotomy for failure of peritoneal drainage in necrotizing enterocolitis in infants with extremely low birth weight. J Pediatr Surg 2000; 35: 856859.

25-Takamatsu H, Akiyama H, Ibara S, Seki S, Kuraya K, Ikenoue T: Treatment for necrotizing enterocolitis perforation in the extremely premature infant (weighing less than 1,000 g). J Pediatr Surg 1992; 27: 741-743.

26-Rees C M, Hall N J, Eaton S, Pierro A: Surgical strategies for necrotizing enterocolitis: A survey of practice in the United Kingdom. Arch Dis Child Fetal 
Neonatal Ed 2005; 90: 152-155. 27-Rees CM, Kiely EM, Wade AM, Pierro A: Peritoneal drainage or laparotomy for neonatal bowel perforation? A randomized controlled trial. Ann Surg 2008; 248: 4451.

28-Romero RM, García-Casillas MA, Matute JA, Barrientos G, Zamora A: Role of peritoneal drainage in very low birth weight with enterocolitis. Cir Pediatr 2005; 18: 88-92.
29-Cass DL, Brandt ML, Patel DL, Nuchtern JG, Minifee PK, Wesson DE: Peritoneal drainage as definitive treatment for neonates with isolated intestinal perforation. $J$ Pediatr Surg 2000; 35: 1531-1536. 30-Blakely ML, Lally KP, McDonald S, et al: Postoperative outcomes of extremely low birth-weight infants with necrotizing enterocolitis or isolated intestinal perforation: A prospective cohort study by the NICHD Neonatal Research Network. Ann Surg 2005; 241: 984-994. 